PACS 75.10.Jm

\title{
LOCAL PAIRING IN THE ATTRACTIVE HUBBARD MODEL
}

\author{
T.Di Matteo, F.Mancini, S.Marra \\ INFM e Dipartimento di Fisica Teorica e S.M.S.A. \\ Universitá di Salerno , I-84140 Baronissi(SA), Italy
}

Received September 12, 1995

The Hubbard model with on-site attractive interaction (negative$\mathrm{U}$ Hubbard model) is studied by means of the composite operator method in the static approximation for the case of half filling. At zero temperature, when the strength of the attractive interaction equals the band width, the system exhibits a phase transition to a pair state, where all the electrons are locally paired. The temperature $T_{p}$ which controls the cross over to the pair state is calculated as a function of $U$. The double occupancy and the spin magnetic susceptibility $\chi_{0}$ are calculated for various values of $U$ and $T$. For strong attractive interaction $\chi_{0}$ is strongly depressed, and tends to zero as $T \rightarrow T_{P}$.

\section{Introduction}

An interesting problem in the physics of strongly correlated electron systems is the study of systems where the formation of electron pairs is possible in the real space: local electron pairing. This problem has been extensively studied both from experimental and theoretical point of view; for an excellent and exhaustive review we refer the reader to the work of Ref. 1 . The formation of local pairs requires an effective attraction which overcomes the repulsive Coulomb potential. There are several mechanisms which can induce a short range attraction among the electrons (coupling of the electrons with the lattice, coupling of the electrons with quasibosonic excitations, chemical mechanisms, etc.) and many physical systems exhibit properties related to the existence of local electrons pairs (superconductors, transition metal oxides, alternating-valence compounds, etc.). A simple model to investigate this problem is given by the extended Hubbard model

$$
H=\sum_{i j} t_{i j} c^{\dagger}(i) \cdot c(j)+U \sum_{i} n_{\uparrow}(i) n_{\downarrow}(i)+1 / 2 \sum_{i j} W_{i j} n(i) n(j)-\mu \sum_{i} n(i) .
$$

The variable i stands for the lattice vector $\mathbf{R}_{\mathbf{i}} \cdot\left\{c(i), c^{\dagger}(i)\right\}$ are annihilation and creation operators of c-electrons at site $i$, in the spinor notation:

$$
c=\left(\begin{array}{c}
c_{\uparrow} \\
c_{\downarrow}
\end{array}\right), \quad \quad c^{\dagger}=\left(c_{\uparrow}^{\dagger}, c_{\downarrow}^{\dagger}\right),
$$

(c) T.Di Matteo, F.Mancini, S.Marra, 1996

ISSN 0452-9910. Condensed Matter Physics 1996 No 8 (109-117) 
$t_{i j}$ is the transfer integral; the $U$ - and $W$-terms describe on-site and intersite interactions, respectively; $n(i)=n_{\uparrow}(i)+n_{\jmath}(i)$, with $n_{\sigma}(i)=c_{\sigma}^{\dagger}(i) c_{\sigma}(i)$ being the number operator of electrons with spin $\sigma ; \mu$ is the chemical potential. This model has been extensively studied for various lattice dimensions by means of different theoretical approaches and numerical analysis; appropriate references can be found Ref.1. Many analytic and numerical methods have been developed for the study of strongly correlated electron systems. In the last years we have been developing a method of calculation, the composite operator method, based on the consideration that in strongly correlated systems it is not convenient to develop approximate solutions around the original particles, since in the observation their properties are strongly modified by the interactions. Instead, we develop approximate solutions starting from a convenient set of composite fields, which correspond to the original fields dressed by interactions and compute their properties by a self consistent calculations. The method has been applied to different models: p-d model [2], Heisenberg model [3], Hubbard model [4], Kondo-Heisenberg model [5]. In this article we shall consider the case of the Hubbard model with on-site attraction. In Section 2 we derive the explicit expression for the Green's function, in the static approximation. In Section 3 we present some results for the double occupancy and the spin magnetic susceptibility for the case of half filling. By analytic method we can show that at zero temperature the system exhibits a phase transition to a pair state, where all the electrons are locally paired in a singlet state; the critical value $U_{c}$ for the interaction potential at which the phase transition occurs is equal to $U_{c}$ $=-8 t, 8 t$ being the band width.

\section{The negative-U Hubbard model}

Systems where the induced local attraction overcomes the on-site Coulomb repulsion can be conveniently described by the so-called negative-U Hubbard model, defined by the following Hamiltonian

$$
H=\sum_{i j} t_{i j} c^{\dagger}(i) \cdot c(j)+U \sum_{i} n_{\uparrow}(i) n_{\downarrow}(i)-\mu \sum_{i} c^{\dagger}(i) \cdot c(i)
$$

with $U<0$. We shall study a squared two-dimensional lattice in the nearestneighbour approximation; the hopping matrix has the expression

$$
\begin{gathered}
t_{i j}=-4 t \alpha_{i j}=-4 t \frac{1}{N} \sum_{\mathbf{k}} e^{i \mathbf{k} \cdot\left(\mathbf{R}_{i}-\mathbf{R}_{j}\right)} \alpha(\mathbf{k}), \\
\alpha(\mathbf{k})=\frac{1}{2}\left[\cos \left(k_{x} a\right)+\cos \left(k_{y} a\right)\right],
\end{gathered}
$$

$a$ being the lattice constant. In the framework of the composite operator method [4], we introduce the field

$$
\psi(i)=\left(\begin{array}{c}
\xi(i) \\
\eta(i)
\end{array}\right)
$$

where $\xi(i)$ and $\eta(i)$ are the Hubbard operators

$$
\xi_{\sigma}(i)=c_{\sigma}(i)\left[1-n_{-\sigma}(i)\right],
$$




$$
\eta_{\sigma}(i)=c_{\sigma}(i) n_{-\sigma}(i)
$$

By means of Hamiltonian (2.1) one obtains the equation of motion:

$$
i \frac{\partial}{\partial t} \psi(i)=j(i)
$$

where the source term $\mathrm{j}(\mathrm{i})$ has the expression

$$
j(i)=\left(\begin{array}{c}
-\mu \xi(i)-4 t\left[c^{\alpha}(i)+\pi(i)\right] \\
-(\mu-U) \eta(i)+4 t \pi(i)
\end{array}\right)
$$

with

$$
\begin{aligned}
c^{\alpha}(i) & \equiv \sum_{j} \alpha_{i j} c(j), \\
\pi(i) & =\frac{1}{2} \sigma^{\mu} n_{\mu}(i) c^{\alpha}(i)+c(i)\left[c^{\alpha^{\dagger}}(i) \cdot c(i)\right] .
\end{aligned}
$$

Let us introduce the retarded thermal Green's function

$$
S(i, j)=\left\langle R\left[\psi(i) \psi^{\dagger}(j)\right]\right\rangle,
$$

where the bracket indicates the thermal average; by decomposing j(i) as:

$$
j(i)=\epsilon(-i \vec{\nabla})+\delta j(i)
$$

with the constrain

$$
\left\langle\left\{\delta j_{n}(i), \psi_{m}(j)\right\}\right\rangle_{E . T}=0
$$

the Fourier transform of $S(i, j)$ takes the expression [4]

$$
S(\mathbf{k}, \omega)=\frac{1}{\omega-\epsilon(\mathbf{k})-\Sigma(\mathbf{k}, \omega)} I(\mathbf{k}),
$$

where the kinetic $\epsilon(\mathbf{k})$ and the dynamical $\Sigma(\mathbf{k}, \omega)$ parts of the self energy are given by

$$
\begin{aligned}
\epsilon(\mathbf{k}) & =m(\mathbf{k}) I^{-1}(\mathbf{k}), \\
\Sigma(\mathbf{k}, \omega) & =M(\mathbf{k}, \omega) S^{-1}(\mathbf{k}, \omega),
\end{aligned}
$$

with

$$
\begin{aligned}
I(\mathbf{k}) & =\left\langle\left\{\psi(i), \psi^{\dagger}(i)\right\}\right\rangle_{F . T}, \\
m(\mathbf{k}) & =\left\langle\left\{j(i), \psi^{\dagger}(j)\right\}\right\rangle_{F . T}, \\
M(\mathbf{k}, \omega) & =\left\langle R\left[\delta j(i), \psi^{\dagger}(j)\right]\right\rangle_{F . T} .
\end{aligned}
$$

In order to explicitly calculate the Green's function we must introduce some approximation. In this article we compute $S(\mathbf{k}, \omega)$ by neglecting the dynamical part $\Sigma(\mathbf{k}, \omega)$. As shown in our previous works [4], this approximation seems quite reasonable, since we can reproduce with quite good accuracy most of the results obtained by means of numerical analysis. Then, we need the knowledge of the normalization matrix given by (2.17) and of the 
m-matrix, defined by (2.18). The attractive interaction will favour the formation of local singlet pairs and contrast the establishment of a magnetic order [6]; therefore, we restrict the analysis to the paramagnetic phase and we have

$$
\left\langle n_{\uparrow}(i)\right\rangle=\left\langle n_{\downarrow}(i)\right\rangle=n / 2 .
$$

A straightforward calculation gives:

$$
\begin{aligned}
& I(\mathbf{k})=\left(\begin{array}{cc}
I_{11} & 0 \\
0 & I_{22}
\end{array}\right) \\
&=\left(\begin{array}{cc}
1-\frac{n}{2} & 0 \\
0 & \frac{n}{2}
\end{array}\right), \\
& \epsilon(\mathbf{k})=m(\mathbf{k}) I^{-1}(\mathbf{k}) \\
&=\left(\begin{array}{cc}
\epsilon_{11}(\mathbf{k}) & \epsilon_{12}(\mathbf{k} \\
\epsilon_{21}(\mathbf{k} & \epsilon_{22}(\mathbf{k}
\end{array}\right) \\
&=\left(\begin{array}{ll}
m_{11}(\mathbf{k}) I_{11}^{-1}(\mathbf{k}) & m_{12}(\mathbf{k}) I_{22}^{-1}(\mathbf{k}) \\
m_{21}(\mathbf{k}) I_{11}^{-1}(\mathbf{k}) & m_{22}(\mathbf{k}) I_{22}^{-1}(\mathbf{k})
\end{array}\right), \\
& m_{11}(\mathbf{k})=\mu\left(\frac{n}{2}-1\right)-4 t[\Delta+\alpha(\mathbf{k})(1-n+p)], \\
& m_{12}(\mathbf{k})= m_{21}(\mathbf{k})=4 t\left[\Delta-\alpha(\mathbf{k})\left(\frac{n}{2}-p\right)\right], \\
& m_{22}(\mathbf{k})= {[-\mu+U] \frac{n}{2}-4 t[\Delta+\alpha(\mathbf{k}) p], }
\end{aligned}
$$

where

$$
\begin{aligned}
\Delta & \equiv\left\langle\xi^{\alpha}(i) \xi^{\dagger}(i)\right\rangle-\left\langle\eta^{\alpha}(i) \eta^{\dagger}(i)\right\rangle, \\
p & \equiv \frac{1}{4}\left\langle n_{\mu}^{\alpha}(i) n_{\mu}(i)\right\rangle-\left\langle\left[\xi_{\uparrow}(i) \eta_{\downarrow}(i)\right]^{\alpha} \eta_{\downarrow}^{\dagger}(i) \xi_{\uparrow}^{\dagger}(i)\right\rangle .
\end{aligned}
$$

By means of these results, the matrix elements of the Green's function (2.14) have the following expressions

$$
\begin{aligned}
\left\langle R\left[\xi(i) \xi^{\dagger}(j)\right]\right\rangle_{F, T} & =S_{11}(\mathbf{k}, \omega) \\
& =\frac{I_{11}}{4 Q(\mathbf{k})}\left[\frac{2 Q(\mathbf{k})+\Delta \Sigma(\mathbf{k})}{\omega-E_{1}(\mathbf{k})+i \eta}+\frac{2 Q(\mathbf{k})-\Delta \Sigma(\mathbf{k})}{\omega-E_{2}(\mathbf{k})+i \eta}\right] \\
\left\langle R\left[\xi(i) \eta^{\dagger}(j)\right]\right\rangle_{F, T} & =\left\langle R\left[\eta(i) \xi^{\dagger}(j)\right]\right\rangle_{F, T}=S_{12}(\mathbf{k}, \omega) \\
& =\frac{m_{12}}{2 Q(\mathbf{k})}\left[\frac{1}{\omega-E_{1}(\mathbf{k})+i \eta}-\frac{1}{\omega-E_{2}(\mathbf{k})+i \eta}\right] \\
\left\langle R\left[\eta(i) \eta^{\dagger}(j)\right]\right\rangle_{F, T} & =S_{22}(\mathbf{k}, \omega)= \\
& =\frac{I_{22}}{4 Q(\mathbf{k})}\left[\frac{2 Q(\mathbf{k})-\Delta \Sigma(\mathbf{k})}{\omega-E_{1}(\mathbf{k})+i \eta}+\frac{2 Q(\mathbf{k})+\Delta \Sigma(\mathbf{k})}{\omega-E_{2}(\mathbf{k})+i \eta}\right] .
\end{aligned}
$$

$E_{1}(\mathbf{k})$ and $E_{2}(\mathbf{k})$ are the energy spectra of the elementary excitations:

$$
E_{1}(\mathbf{k})=R(\mathbf{k})+Q(\mathbf{k}), \quad E_{2}(\mathbf{k})=R(\mathbf{k})-Q(\mathbf{k}) .
$$


The definitions are

$$
\begin{gathered}
R(\mathbf{k})=\frac{1}{2}[-2 \mu+U]-\frac{2 t}{I_{11} I_{22}}\left\{\Delta+\alpha(\mathbf{k})\left[p+(1-n) I_{22}\right]\right\} \\
Q(\mathbf{k})=\frac{1}{2} \sqrt{U^{2}+\frac{m_{12}^{2}(\mathbf{k})}{I_{11}^{2} I_{22}^{2}}-2 U \frac{(1-n) m_{12}(\mathbf{k})}{I_{11} I_{22}}}, \\
\Delta \Sigma(\mathbf{k})=-U+\frac{(1-n) m_{12}(\mathbf{k})}{I_{11} I_{22}}
\end{gathered}
$$

The expressions (2.28)-(2.30) for the matrix elements contain three parameters, $\mu, \Delta$ and $p$, which must be self consistently calculated. The chemical potential $\mu$ is determined in terms of the particle density $n$ by means of the equation

$$
n=\left\langle c^{\dagger} \cdot c\right\rangle=2\left[1-\left\langle\xi(i) \xi^{\dagger}(i)\right\rangle-\left\langle\eta(i) \eta^{\dagger}(i)\right\rangle\right] .
$$

The amplitude $\Delta$ is expressed in terms of matrix elements of $S(i, j)$, where $j$ is the first nearest neighbouring site to $i$, as

$$
\Delta \equiv\left\langle\xi^{\alpha}(i) \xi^{\dagger}(i)\right\rangle-\left\langle\eta^{\alpha}(i) \eta^{\dagger}(i)\right\rangle
$$

The parameter $p$, defined by (2.27), is related to intersite charge, spin and pair fluctuations and is computed by means of the equation

$$
\left\langle\xi(i) \eta^{\dagger}(i)\right\rangle=0,
$$

which recovers the Pauli principle. Equations (2.35)- (2.37) constitute a set of coupled equations which fix in a self consistent way the three parameter $\mu, \Delta$ and $p$. Once these quantities are evaluated, the Green's function can be computed; then, the properties of the system can be computed. For example, one quantity which characterizes the system is the double occupancy

$$
D=\left\langle n_{\uparrow} n_{\downarrow}\right\rangle=\frac{n}{2}-S_{22}(i, j)
$$

which gives the average number of sites occupied by two electrons. Another important quantity which describes the magnetic properties is given by the spin magnetic susceptibility; in the present approximation this quantity is given by [7]

$$
\begin{aligned}
\chi(\mathbf{k}, \omega) & =\frac{n(2-n)}{n^{2}-n-2 D}\left[I _ { 1 1 } ^ { - 1 } \left(Q_{1111}^{R}+2 Q_{1112}^{R}\right.\right. \\
& \left.\left.+Q_{1212}^{R}\right)+I_{22}^{-1}\left(Q_{1212}^{R}+2 Q_{1222}^{R}+Q_{2222}^{R}\right)\right]
\end{aligned}
$$

where $Q_{\alpha \beta \gamma \delta}^{R}$ is the retarded part of

$$
Q_{\alpha \beta \gamma \delta}(\mathbf{k}, \omega)=i \Omega \int \frac{d^{2} p d \Omega}{(2 \pi)^{3}} S_{\alpha \beta}(\mathbf{k}+\mathbf{p}, \omega+\Omega) S_{\gamma \delta}(\mathbf{p}, \Omega) .
$$

$S_{\alpha \beta}(\mathbf{k}, \omega)$ being the elements of the causal thermal Green's function. 


\section{Half filling and pair state}

In a previous work [8] we have studied the system for various values of the particle density; the results are in good agreement with the data obtained by numerical analysis [9]. In this article we concentrate on the case of half filling. By analytic methods it is possible to show that at zero temper-

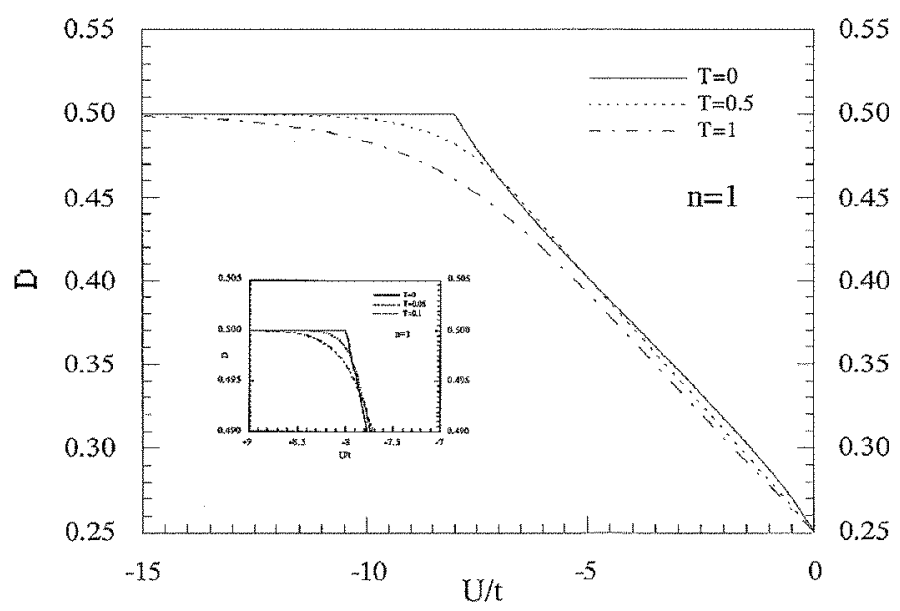

Figure 1. The double occupancy $D$ is reported as a function of the potential strength $U / t$ for various values of the reduced temperature $K_{B} T / t$.

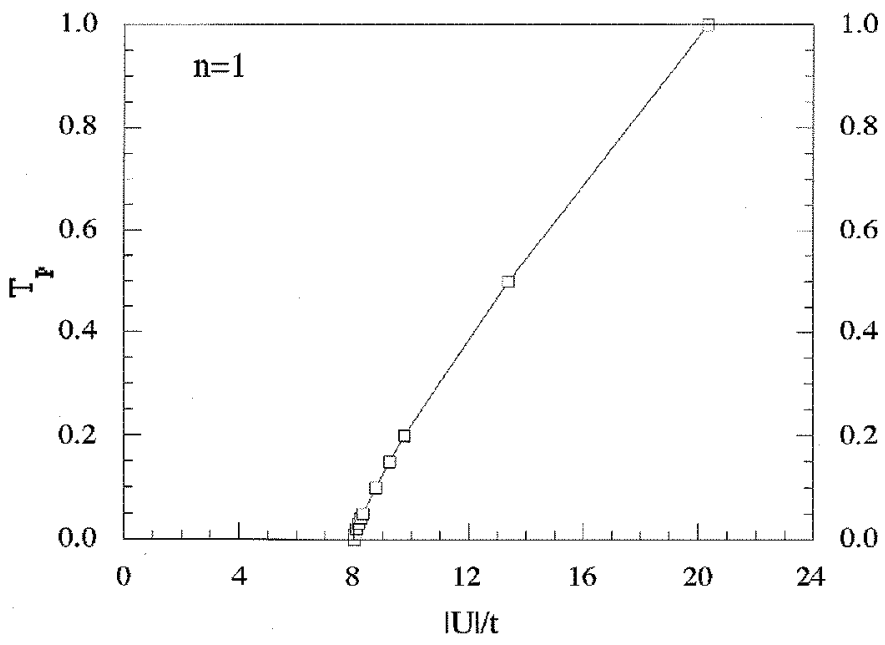

Figure 2. The "critical" temperature $T_{P}$ which characterizes the "phase" transition to the pair state is reported as a function of the potential strength $U / t$.

ature the system exhibits a phase transition: there is a critical value of the interaction $U_{c}=-8 t$, such that for $|U|>8 t$ the system is in a pair state characterized by the fact that all electrons are locally paired; the double occupancy is equal to $1 / 2$ and the uniform static spin magnetic susceptibility 
$\chi_{0} \equiv \chi(0,0)$ vanished. Details of calculations will be presented elsewhere, in this note we limit ourselves to present the results of calculations. In figure 1 the double occupancy is reported as a function of the interaction for different values of temperature. In the limit of vanishing interaction the double

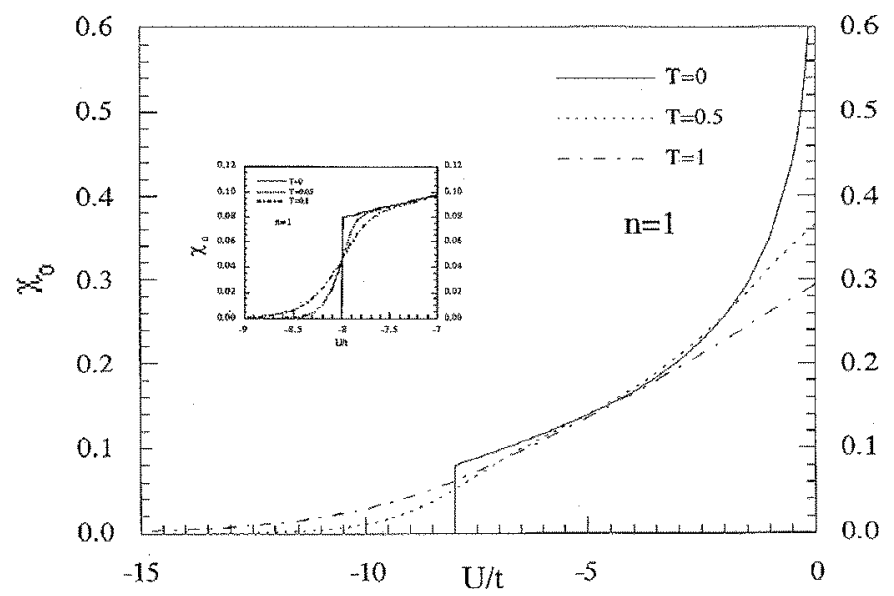

Figure 3. The uniform static spin magnetic susceptibility $\chi(0)$ is reported as a function of the potential strength $U / t$ for various values of the reduced temperature $K_{B} T / t$.

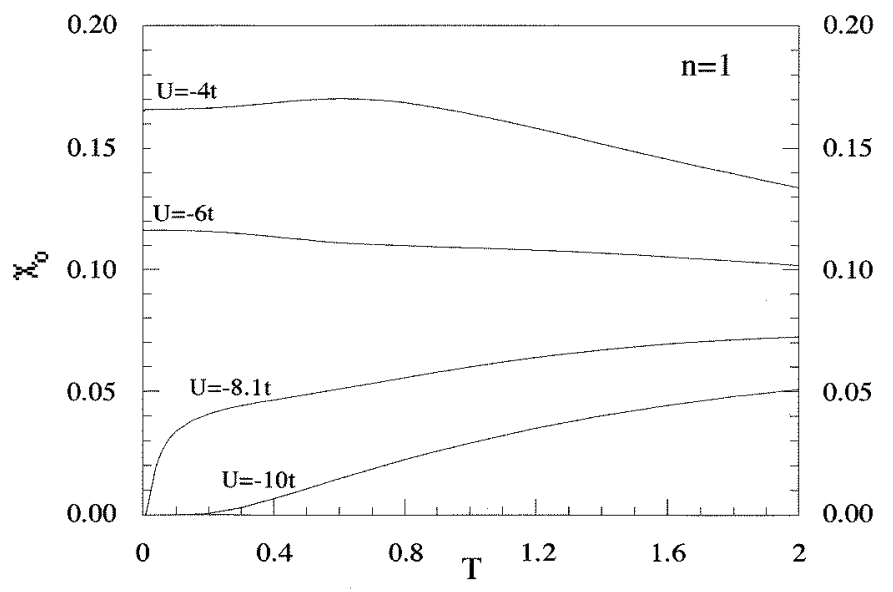

Figure 4. The uniform static spin magnetic susceptibility $\chi(0)$ is reported as a function of the reduced temperature $K_{B} T / t$ for various values of the potential strength $U / t$.

occupancy is equal to $1 / 4$; by increasing $|U|$ local pairing is favoured and $D$ increases; for zero temperature $D$ reaches the maximum value $D=1 / 2$ at $U=-8 t$; for finite temperature the thermal fluctuations tends to break the local pairs and $D$ tends asymptotically to the maximum value only in the limit of $|U| \rightarrow \infty$. To study the transition to the pair state, for a fixed potential we have calculated the value of the temperature $T_{P}$ such that $1 / 2-D<10^{-4}$; the results are reported in figure 2 where $T_{P}$ is given as 
a function of $|U|$. The line in the plane $(T, U)$ indicates a crossover to the regime of pair state. Approximately, we might take $T_{P}$ as the critical temperature for the breaking of electron pairs. From mean field results [1] we expect that below $T_{P}$ there is another critical temperature which separates the phase of uncorrelated pairs from a long-range ordered phase (superconductivity or/and charge density wave). The possible existence of an ordered phase in which the pairs are correlated is currently under investigation by means of COM.

The uniform static spin magnetic susceptibility $\chi_{0}$ is reported in figure 3 as a function of $U$ for different temperatures. At zero temperature $\chi_{0}$ goes abruptly to zero when $U$ reaches the value $U=-8 t$, in correspondence to the phase transition to the pair state. In this phase the electrons are all paired in a singlet state and of course there is no magnetic answer. When $T \neq 0, \chi_{0}$ goes asymptotically to zero only in the limit of infinite $U$, but for $T<T_{P} \chi_{0}$ is practically zero, as shown in figure 3. Generally, the spin magnetic susceptibility is strongly depressed by the attractive interaction. This can be clearer seen in figure 4 where $\chi_{0}$ is given as a function of temperature for different values of the interaction. For weak attraction the susceptibility has a Heisenberg-like dependence, as in the case of repulsive attraction [7], though quite depressed. For strong attraction the dependence of $\chi_{0}$ on $T$ is completely different $: \chi_{0} \rightarrow 0$ as $T \rightarrow T_{P}$ and increases with $T$. This is due to the fact that for strong attraction the electrons are practically all singlet paired and can give a non-zero contribution to the susceptibility only when the thermal fluctuations break the pair.

\section{References}

[1] Micnas R., Ranninger J., Robaszkiewicz R. Superconductivity in narrow-band systems with local nonretarded attractive interactions. // Rev. of Mod. Phys., 1990, vol. 62, p. 113.

[2] Ishihara I., Matsumoto H., Odashima S., Tachiki M., Mancini F. Meanfield analysis in the p-d model of oxide Superconductors. // Phys. Rev. B, 1994, vol. 49, p. 1350;

Mancini F., Marra S., Villani D., Matsumoto H. Local magnetic moment in the two-dimensional reduced p-d model. Preprint University of Salerno (1995).

[3] Allega A.M., Odashima S., Matsumoto H., Mancini F. Static and dynamical spin susceptibility in 2D antiferromagnetic Heisenberg model. // Physica C, 1994, vol. 235-240, p. 2229.

[4] Mancini F., Marra S., Matsumoto H. Doping dependence of on-site quantities in the two-dimensional Hubbard model. // Physica C, 1995, vol. 244, p. 49;

Mancini F., Marra S., Matsumoto H. Energy and chemical potential in the two-dimensional Hubbard model. // Physica C, 1995, vol. 250, p. 184.

[5] Matsumoto H., Allega A.M., Odashima S., Mancini F. Metal-insulator transition in Kondo-Heisenberg model of oxide superconductors. // Physica C , 1994, vol. 235-240, p. 2227.

[6] Lieb E.H. Two theorems on the Hubbard model. // Phys. Rev. Lett., 1989, vol. 62, p. 1201.

[7] Mancini F., Marra S., Matsumoto H. Spin magnetic susceptibility in the two- dimensional Hubbard model. // Physica C (in press). 
[8] Di Matteo T., Mancini F., Marra S., Matsumoto H. Analysis of the twodimensional negative- U Hubbard model by composite operator method. (Preprint July 1995). Submitted to Physica B.

[9] Randeria M., Trivedi N., Moreo A., Scalettar R.T. Pairing and spin gap in the normal state of short coherence length superconductors. // Phys. Rev. Lett., 1992, vol. 69, p. 2001.

\title{
ЛОКАЛЬНЕ СПАРЮВАННЯ В ПРИТЯГУВАЛЬНІЙ МОДЕЛІ ХАББАРДА
}

\author{
Т. ді Маттео, Ф.Манчіні, С.Марра
}

\begin{abstract}
Досліджується модель Хаббарда з одноцентровою притягувальною взаємодією ( $U$-негативна модель Хаббарда) з використанням методу композитного оператора у статичному наближенні для випадку напівзаповнення. При нульовій температурі, коли сила притягувальної взаємодії рівна ширині смуги, система зазнає фазового переходу до парного стану, коли всі електрони є локально спарені. Температура $T_{p}$, що контролює перехід до парного стану, обчислюеться як функція $U$. Подвійна заселеність та спінова магнітна сприятливість $\chi_{0}$ обчислюються для різних значень $U$ та $T$. Для сильно притягувальної взаємодії $\chi_{0}$ сильно подавляється й прямуе до нуля при $T \rightarrow T_{p}$.
\end{abstract}

